\title{
Correlation Between Immunohistochemical Expression Of S100A4 With Histopathological Grading And Molecular Subtype Classification In Non Specifik Invasive Breast Cancer
}

\author{
Hengky Ardian ${ }^{\mathrm{a}}$, T.Ibnu Alferally ${ }^{\mathrm{b}}$, Delyuzar ${ }^{\mathrm{c}}$, Joko S Lukito ${ }^{\mathrm{d}}$, Soekimin ${ }^{\mathrm{e}}, \mathrm{T}$ \\ Kemala $\operatorname{Intan}^{\mathrm{f}}$ \\ Department of Anatomik Pathology. Faculty of Medicine, \\ Universitas Sumatera Utara, Medan, Indonesia \\ Hengkyardian99@yahoo.co.id
}

\begin{abstract}
Background: Breast carcinoma is a malignancy that occurs from breast cells that grow and continue to proliferate uncontrollably. S100A4 has the potential to increase tumor metastasis in preexisting tumorigenic breast cancer. S100A4 protein expression level is associated with poor prognosis in breast cancer.

Objective: To analyze the relationship between immunohistochemical expression of S100A4 with histopathological grading and molecular subtypes in invasive breast carcinoma of no special type.

Methods: Formalin-fixed tissue paraffin blocks from 42 invasive breast carcinomas of no special type were used as research samples and S100A4 immunohistochemical staining was assessed. The relationship between S100A4 expression and histopathological grading and molecular subtypes was analyzed by statistical tests using the SPSS program.

Results: Based on the histopathological grade, grade 3 was 18 samples (42.9\%), grade 1 was 10 samples (23.8\%), and grade 1I was 14 samples (33.3\%). Based on the molecular subtype, Luminal A as many as 16 samples (38.1\%), HER2-enriched as many as 12 samples (28.6\%), Luminal B as many as 12 samples $(28.6 \%)$, and Triple-negative as many as 2 samples $(4.8 \%)$ ). Immunohistochemical expression of S100A4 was positive in as many as 33 samples (78.6\%). Conclusion: There is a significant relationship between the immunohistochemical expression of S100A4, either with histologic grading or with molecular subtypes in Invasive Breast Carcinoma of No Special Type (NST) $\mathrm{p}(0.034)<0.05$ and $\mathrm{p}(0.039)<0.05)$
\end{abstract}

Keywords: Invasive breast carcinoma of NST, histological grading, molecular subtype

\section{Introduction}

One of the most important health problems in women in the world is breast cancer, where more than one million new cases of breast cancer are diagnosed worldwide each year, and about a third of all cancers are in women. The incidence of breast cancer in the United States and Europe is twice that of Asian countries. According to data from the Global Burden Cancer (GLOBOCAN), and the International Agency for Research on Cancer (IARC), one in every 9 women in developed countries and one in every 20 women in less developed areas has a risk of developing breast cancer. The agestandardized incidence rate (ASIR) every year increases by 29 percent in the world.[1,2]

The protein product of the gene is called the metastasis-inducing protein. One such gene/protein is S100A4, a member of the S100-binding protein family. Although S100A4 cannot 
induce tumor formation directly, it can stimulate steps in the metastatic cascade in the model rodent system by combining oncogenes such as RasVal12 and neu. Increased S100A4 can trigger several biological functions, including cell migration, invasion, extracellular matrix remodeling, and angiogenesis. However, it is not known that the biologically relevant molecular events are triggered by S100A4 in mammalian cells.[7]

\section{Material and Methods}

Researchers examined 42 samples from invasive breast carcinoma at $\mathrm{H}$. Adam Malik Hospital Medan which were diagnosed histopathologically by hematoxylin-eosin (HE) staining. This study is an analytical study with a cross-sectional approach to assessing the immunohistochemical characteristics and expression of S100A4, from January 2021 to October 2021. This study was approved by the Committee for Conducting Health Research Ethics, Faculty of Medicine, the Universitas Sumatera Utara with the number: 456/KEP/USU /2021.

This study displays the characteristics of the sample, namely age, histopathological subtype, histopathological grading, molecular subtype, and S100A4 expression. The sample used is a sample that fits the inclusion and exclusion criteria. Inclusion criteria were all paraffin slides and blocks derived from post-mastectomy breast tissue diagnosed as histopathologically representative invasive breast carcinoma of no special type (NST) after hematoxylin-eosin (HE) staining at H. Adam Malik Hospital Medan and have data medical records which include ER, PR, HER2, and Ki 67 immunohistochemical examination data in invasive breast carcinoma patients. Exclusion criteria were paraffin slides and blocks derived from core biopsy and lumpectomy of breast tissue diagnosed histopathologically invasive breast carcinoma, paraffin slides and blocks that could not be re-evaluated or re-cut for examination with S100A4 Immunohistochemistry, and ER PR, HER2 immunohistochemical examination data., and the incomplete Ki-67. The immunohistochemical expression of S100A4 in this study was categorized into negative and positive. He is said to be negative if the expression of S100A4 does not appear brown. And it is said to be positive if the expression of S100A4 looks brown.

\section{Result}

This study involved 42 samples diagnosed histopathologically as invasive breast carcinoma that had met the inclusion criteria and exclusion criteria were collected until the number of samples was sufficient based on the minimal sample size. All samples were post-mastectomy preparations at $\mathrm{H}$. Adam Malik Hospital Medan. Active data of molecular subtypes (ER, PR, HER2, Ki-67) are secondary data from medical record data (pathology archive). In the study, the youngest patient. For the age group, the most common were in the age group 35-49 years and the age group 50-64 years each with 15 cases (35.7\%), the age group 60 years while the age group $<35$ years the least, namely 4 cases $(9.5 \%)$ 
Table 1. Frequency distribution of characteristics of invasive breast carcinoma of no Special type

$\begin{array}{lcc}\text { Sample characteristics } & \text { Total (n) } & \text { Percenta } \\ \begin{array}{l}\text { Age (years) } \\ <35\end{array} & 4 & 9,5 \\ 35-49 & 16 & 38,1 \\ 50-64 & 15 & 35,7 \\ \geq 60 & 7 & 16,7 \\ \text { Grading } & & \\ \text { Grade 1 } & 10 & 23,8 \\ \text { Grade 2 } & 14 & 33,3 \\ \text { Grade 3 } & 18 & 42,9 \\ & & \\ \text { Molecular subtype } & 16 & 38.1 \\ \text { Luminal A } & 12 & 28.6 \\ \text { Luminal B } & 12 & 28.6 \\ \text { HER2-enriched } & 2 & 4.8 \\ \text { Tripel-negative } & & \\ \text { S100A4 expression } & 9 & 21.4 \\ \text { Negative } & 33 & 78.6 \\ \text { Positive } & & \end{array}$

Based on Table 1 above, the most common histopathological grading was grade 3 with 18 cases (42.9\%), and the least encountered was grade 1 with 10 cases $(23.8 \%)$. The most common molecular subtype was luminal A with 16 cases $(38.1 \%)$. Meanwhile, the least triple-negative molecular subtypes were found, namely 2 cases $(4.8 \%)$. The immunohistochemical expression of S100A4 displayed in the cytoplasm of tumor cells was found to be negative in 9 cases $(21.4 \%)$. While positive expression was found in 33 cases $(78.6 \%)$

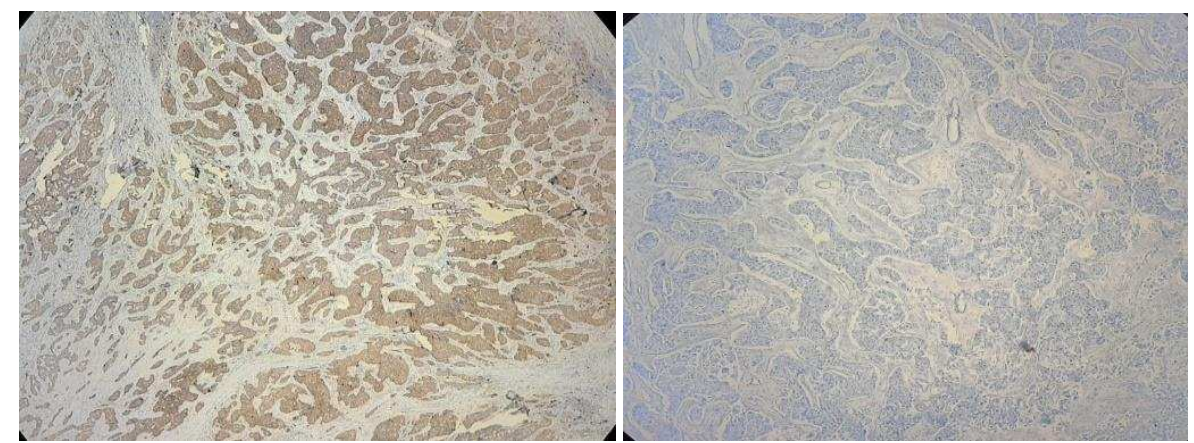

Figure 1. S100A4 immunohistochemical staining in invasive breast carcinoma A. Positive S100A4 expression. B. Negative S100A4 expression. 
Table 2. Relationship of S100A4 Immunohistochemical Expression with Histopathological Grading in Invasive Breast Carcinoma of No Special Type

S100A4

Immunohistochemical Expression

Negative

Positive

\section{Histopathology grading}

\begin{tabular}{ccccccccc}
\multicolumn{2}{c}{ Grade I } & \multicolumn{2}{c}{ Grade $\mathbf{2}$} & \multicolumn{2}{c}{ Grade 3 } & \multicolumn{2}{c}{ Total } & p- \\
$\mathbf{n}$ & $\mathbf{\%}$ & $\mathbf{n}$ & $\mathbf{\%}$ & $\mathbf{n}$ & $\mathbf{\%}$ & $\mathbf{n}$ & $\boldsymbol{\%}$ & \\
0 & 0 & 6 & 66,7 & 3 & 33,3 & 9 & 100,0 & \\
10 & 30,3 & 8 & 24,2 & 15 & 45,5 & 33 & 100,0 & 0,034 \\
10 & & 14 & & 18 & & 42 & & \\
\end{tabular}

Total

*Pearson Chi-Square

From the results of statistical test analysis, it was found that there were 33 samples with positive S100A4 immunohistochemical expression who had grade III as many as 15 people (45.5\%). The results of the Pearson Chi-Square statistical test showed that there was a significant relationship between the expression of S100A4 immunohistochemistry and histopathological grading in Invasive Breast Carcinoma of No Special Type with p-value $(0.034)<0.05$. This means that the positive immunohistochemical expression of S100A4 is likely to show a higher grade.

Table 3. Relationship of S100A4 Immunohistochemical Expression with Classification of Molecular Subtypes in Invasive Breast Carcinoma of No Special Type

S100A4

\section{Molecular Subtypes}

\begin{tabular}{lccccccccccc}
$\begin{array}{l}\text { Immunohistochemica } \\
\quad \text { I Expression }\end{array}$ & Luminal A & Luminal B & \multicolumn{2}{c}{ HER2- } & \multicolumn{2}{c}{ Triple- } & Total & P \\
Negative & $\mathbf{n}$ & $\mathbf{\%}$ & $\mathbf{n}$ & $\mathbf{\%}$ & $\mathbf{n}$ & $\mathbf{\%}$ & $\mathbf{n}$ & $\mathbf{\%}$ & $\mathbf{n}$ & $\mathbf{\%}$ & \\
Positive & 0 & 0 & 5 & 55,6 & 3 & 33,3 & 1 & 11,1 & 9 & 100,0 & \\
Total & 16 & 48,5 & 7 & 21,2 & 9 & 27,3 & 1 & 3,0 & 33 & 100,0 & \\
\hline
\end{tabular}

*Pearson Chi-Square

From the analysis of the relationship between the expression of S100A4 immunohistochemistry with the molecular subtype of Invasive Breast Carcinoma of No Special Type, it was found that there were 33 samples with positive immunohistochemical expression of S100A4 who experienced the Luminal A molecular subtype as many as 16 samples (48.5\%). The results of the Pearson Chi-Square statistical test showed that there was a significant relationship between the expression of S100A4 immunohistochemistry with molecular subtypes in Invasive Breast Carcinoma of No Special Type with p-value $(0.039)<0.05$. This means that the positive immunohistochemical expression of S100A4 is likely to indicate a poorer molecular subtype.

\section{Discussion}

According to the World Health Organization (WHO), as many as $13 \%$ of all deaths in Indonesia due to non-communicable diseases are caused by cancer. Breast cancer when first diagnosed in women in Indonesia is often at an advanced stage. Breast cancer can be successfully treated if it is diagnosed at an early stage and the patient makes lifestyle changes. Several previous studies have revealed that most patients with cancer diagnosed at an advanced stage have a poor prognosis.[3] 
Breast cancer and cervical cancer in Indonesia are the types of cancer with the highest prevalence in women. Based on data from Global Cancer Indonesia in 2020, there were 65,858 new cases of breast cancer or around $16.6 \%$ and the mortality rate from breast cancer was 22,430 cases or around 9.6\%.5 Risk factors that cause breast cancer in individuals, which include: age, family history of similar diseases, hormonal factors such as estrogen and androgens, diet, alcohol, and other factors.[4,5]

Breast cancer has different histopathological and biological characteristics so that it shows different treatment responses and therapeutic strategies given. The grouping of breast cancer into molecular subtypes is carried out to determine the type of treatment carried out so that it has more accurate results. Histopathological examination has a significant relationship with prognostication in breast cancer, namely histological subtype, histological grading, lymph node status, estrogen receptor (ER) status, progesterone receptor (PR), and human epidermal growth factor 2 (HER2).[6]

Histological grading of tumors is determined based on the abnormality of tumor cells in the tissue seen using a microscope. The grading of breast cancer is determined based on the assessment of tubular/glandular formation, nuclear pleomorphism, and the number of mitoses. Hormone receptor (HR) is associated with a better prognosis, patients with PR-positive tumors have a good prognosis, similarly, ER-positive tumors are associated with increased life expectancy in patients and also have a higher response to hormone therapy. In contrast, patients with both receptors (ER and PR) negative have a poorer prognosis. DNA microarray clustering. Breast carcinoma is classified into four groups based on the IHC profile, namely ER, PR, and HER2, with positive or negative results.[6,8,9]

Molecular subtypes in breast cancer have long been known to be associated with patient prognosis, with luminal A having the best prognosis and basal-like or triple-negative having the worst prognosis. Tan et al., stated that the heterogeneity of molecular subtypes in triple-negative breast cancer can affect the therapy and level of cancer aggressiveness. Various proteins are essential for the normal functioning of organisms and the proteome, the entirety of the proteins expressed by the genome of a cell or organ, changes according to time and environment. A better understanding of the metastatic process of breast cancer can be made by investigating differences in protein appearance between breast cancer tissues.[10,11,12]

S100A4 is overexpressed in human primary tumors and is associated with early death in patients with various types of metastatic carcinoma, including breast cancer. In the detection of biomarkers of cancer development including invasion and metastasis, experimental studies have identified the S100A4 protein as an important molecular target for the regulation of metastasis. The protein S100A4, a member of the calcium-binding protein family, has been identified as a cytoplasmic protein associated with the actin/myosin cytoskeleton that drives many cellular processes such as cell growth, cell cycle development, differentiation, and transcription. S100A4 regulates many cellular events required for metastasis, such as regulation of angiogenesis, motility, invasion, and cell survival. Human cancer immunohistochemical studies showed significant expression of S100A4 in breast cancer. Studies have focused on the prognostic significance of S100A4 as a marker of metastasis and unfavorable patient outcome.[7,14]

S100A4 consists of alpha and beta chains with a molecular weight of $10-12 \mathrm{Kd}$. It is a small molecule and can pass through the pores of the nucleus without involving active transport mechanisms. S100A4 binds to and inhibits phosphorylation of the C-terminal peptide p53 by protein kinase C. The tumor suppressor protein p53 has also been identified as a protein that interacts with S100A4 and may provide a link between S100A4 and apoptosis.[15]

S100 family proteins have a variety of intracellular and extracellular functions and have been implicated in tumorigenesis and tumor development. The S100 protein is strongly associated with communication between cancer cells and stromal cells, thereby contributing to tumor metastasis. Chen et al.'s study found that S100 promotes migration and invasion of cancer cells by MMP2 through p53dependent transcriptional expression.[14,17]

S100A4 has the potential to increase tumor metastasis in preexisting tumorigenic breast cancer. S100A4 protein expression level is associated with poor prognosis in breast cancer. In addition, 
depletion of S100A4 positive stromal cells significantly reduced the metastatic potential of orthotopic breast tumors without affecting primary tumor growth. Anti-S100A4 monoclonal antibody treatment efficiently reduces the metastatic burden by suppressing $\mathrm{T}$ cell recruitment to the primary tumor site.[16]

\section{Conclusion}

From the results of this study, there is a significant relationship between the expression of S100A4 immunohistochemistry with histopathological grading and subtype in Invasive Breast Carcinoma of No Special Type $(\mathrm{p}(0.034)<0.05)$

\section{Acknowledgment}

We acknowledge all staff and residents of the Department of Pathology Anatomy, Faculty of Medicine, Universitas Sumatera Utara / Haji General Hospital, Malik Medan Medan for all your support and guidance.

\section{Reference}

1. Solikhah, Matahari R., Utami F., Handayani L. and Marwati T., Breast cancer stigma among Indonesian women: a case study of breast cancer patients. BMC Women's Health (2020) 20:116https://doi.org/10.1186/s12905-020-00983

2. Henry NL, Shah PD, Haider I, Freer PE, Jagsi R, and Michael S. Sabelet al: Cancer of the Breast. In: Niederhuber JE, Armitage JO, Doroshow JH, Kastan MB, Tepper JE, eds. Abeloffer Clinical Oncology. 6th ed. Philadelphia, Pa: Elsevier; 2020.

3. World Health Organization, Breast Cancer, Globocan Indonesia 2020. International Agency ResearchCancer

4. Furqan M., Pohan P., Relationship of Histopathology Grading with Molecular Subtypes of Breast Cancer Patients in Haji Adam Malik General Hospital 2016-2018. SCRIPTA SCORE Scientific Medical Journal, Vol. 2, No. 1, Agustus2020

5. Ismail T., Bennett D., Platt-Higgins A., Morteta Al-Medhity, Roger Barraclough, and Philip S. Rudland. S100A4 Elevation Empowers Expression of Metastasis Effector Molecules in Human Breast Cancer. American Association for Cancer Research. November 10, 2016; DOI: 10.1158/0008-5472.CAN-16-1802

6. Soares MC, Rodrigues IJM, Almeida I. , Assunção J. , Monteiro A , and Júnior E., Histopathological And Immunohistochemical Parameters Of Breast Cancer Cases Analyzed In A Reference Laboratory. Mastology2020;30:e20190029

7. Adani-Ife, Amegbor K., Doh K., and Darre T., Breast cancer in Togolese women: immunohistochemistry subtypes. BMC Women's Health (2020) 20:261https://doi.org/10.1186/s12905-020-01130-2

8. Lefebvre C, Bachelot T, Filleron T, Pedrero M., Campone M., Soria J.,et al. Mutational profile of metastatic breast cancers: a retrospective Analysis. PLoSMed 2016;13:e1002201

9. Siraj AK, Beg S, Jehan Z, Prabhakaran S, Ahmed M, Hussain A, et al. ALK alteration is a frequent event in aggressive breast cancers. Breast CancerRes2015;17:127.

10. Tan B, Kanoko M, Tan G, Bachtiar A, Munir D. Dimensional analysis of CD44 high CD24 low and Ki67 in triple-negative breast cancer. Maced J MedSci.2019;7(4):526-528

11. Zhong JM, Li J, Kang AD, Huang S., Liu W., Zhang W., et al. Protein S100-A8: A potential metastasis-associated protein for breast cancer determined via iTRAQ quantitative proteomic and clinicopathological analysis. Oncol Lett. 2018;15(4):5285- 5293

12. Abdelwahab M. and Abdelrahman A. Immunohistochemical expression of Bmi-1, S100A4, and claudin-1 in breast cancer. Egyptian Journal of Pathology 2017,37:82-90 
13. Ismail N., Kaur G., Hashim H. and Hassan M., S100A4 overexpression proves to be an independent marker for breast cancer progression. BioMedCentral. Cancer Cell International 2008, 8:12 doi:10.1186/1475-2867-8-12

14. Chen H, Xu C, Jin Q, Liu Z. S100 protein family in human cancer. Am J Cancer Res. 2014;4:89-115

15. Chen H, Yuan Y, Zhang C, Luo A, Ding F, Ma J et al. Involvement of S100A14 protein in cell invasion by affecting expression and function of matrix metalloproteinase (MMP)-2 via p53-dependent transcriptional regulation. J Biol Chem. 2012; 287:17109-19. 